\title{
Bei Lumbalschmerz nicht gleich ins MRT
}

\author{
Nur rund $44 \%$ der Magnetreso- \\ nanztomografien (MRT), die in der \\ Diagnostik lumbaler Beschwerden \\ angeordnet werden, erfolgen laut \\ einer kanadischen Studie zu Recht.
}

Die Forschergruppe um den Radiologen Derek Emery (Alberta) hat die Angemessenheit von MRT anhand einer Methode beurteilt, die von der US-Denkfabrik RAND (Research and Development) und der University of California, Los Angeles, entwickelt worden ist. Dabei wird ein Expertenpanel von Fachleuten eines Bereichs gebildet, die aufgrund der vorliegenden Daten den Einsatz einer Maßnahme mit einer Punktzahl von 1 bis 9 belegen. Als unangemessen gilt der Einsatz bei ohne Dissens vergebenen Punktzahlen von 1 bis 3, als angemessen bei Punktzahlen von 7 bis 9. Liegt der Median der verteilten Punkte zwischen 4 und 9 oder herrscht Uneinigkeit unter den Experten, wird die Angemessenheit der Maßnahme als fraglich beurteilt. 1000 lumbale MRT und 1000 MRT-Aufnahmen des Schädels, letztere zur Abklärung von Kopfschmerzen, wurden gemäß diesen Vorgaben unter die Lupe genommen. Das Prädikat „angemessen“ erhielten 443 lumbale Tomografien. 285 waren von fraglichem Wert, in 272 Fällen konnten die Experten unisono keinen Nutzen der MRT erkennen. Nur eine Indikation im Lumbalbereich war weitgehend unumstritten. Sie betraf $167 \mathrm{~Pa}-$ tienten, bei denen postoperative Bein- oder Rückenschmerzen bestanden. 160 dieser MRT-Untersuchungen (95,8\%) galten den Fachleuten als adäquat. Bei den sonstigen Anlässen für die MRT-Diagnostik lagen Radikulopathien an der Spitze; auf sie entfielen 29,6\% der MRT im Lumbalbereich.

\section{Hausärzte sind Hauptüberweiser}

Die meisten der Überweisungen zur Lumbal-MRT, insgesamt $61,1 \%$, stammten von Allgemeinärzten. Nur 33,9\% davon galten als adäquat. Den höchsten Anteil an Angemessenheit erzielten Neurochirurgen mit 75,7\%. Andererseits bedeutet dies, dass selbst jede vierte neurochirurgisch veranlasste MRT nicht oder womöglich nicht notwendig war. Ganz anders die Situation bei den MRT im Kopfbereich. Angemessen war der Kernspin-Einsatz hier in $82,8 \%$ der Fälle. Als fraglich wurden $8,2 \%$, als überflüssig $9 \%$ der Untersuchungen eingestuft. Anlass für die MRT waren weit überwiegend nicht näher spezifizierte Zephalgien (43,3\%). Auch hier waren Allgemeinmediziner mit 53,1\% die Hautüberweiser. 10,4\% ihrer Überweisungen zum Kopf-MRT waren unangemessen, die Quote unterschied sich kaum von jenen anderer Fachgruppen. Bei der hohen Zustimmungsrate hinsichtlich der Schädel-MRT ist allerdings zu berücksichtigen, dass die meisten Patienten zuvor bereits eine Computertomografie hinter sich gebracht hatten und also entsprechend vorselektiert waren.

(Robert Bublak)

\section{Hier steht eine Anzeige.}

\author{
黛 Springer
}

